\title{
Chaotic hash function based on circular shifts with variable parameters
}

Yantao $\mathrm{Li}^{1,2, *}, \mathrm{Xiang} \mathrm{Li}^{1}$

${ }^{1}$ College of Computer and Information Sciences, Southwest University, Chongqing 400715, P. R. China

${ }^{2}$ State Key Laboratory for Novel Software Technology, Nanjing University, Jiangsu 210023, P. R. China

*Corresponding author: Yantao Li

Email: yantaoli@foxmail.com, liyantao@live.com, yantaoli@swu.edu.cn (Y. Li) 


\section{Abstract}

We propose a chaotic hash algorithm based on circular shifts with variable parameters in this paper. We exploit piecewise linear chaotic map and one-way coupled map lattice to produce initial values and variable parameters. Circular shifts are introduced to improve the randomness of hash values. We evaluate the proposed hash algorithm in terms of distribution of the hash value, sensitivity of the hash value to slight modifications of the original message and secret keys, confusion and diffusion properties, robustness against birthday and meet-in-the-middle attacks, collision tests, analysis of speed, randomness tests, flexibility, computational complexity, and the results demonstrate that the proposed algorithm has strong security strength. Compared with the existing chaotic hash algorithms, our algorithm shows moderate statistical performance, better speed, randomness tests, and flexibility.

\section{Keywords:}

Chaos, Hash function, Variable parameters, Piecewise linear chaotic map, One-way coupled map lattice

\section{Introduction}

A hash function is a one-way function that can be used to map arbitrary length of digital data to digital data with fixed length. The digital data returned by a hash function are referred to as hash value, or message digest. Hash functions have been used in a wide range of applications, such as integrity protection [1], message authentication [2] and digital signature [3], which have the properties of sensitivity to 
initial conditions, diffusion and confusion, collision resistance. Traditional hash functions such as MD5 and SHA-1 are mainly based on logical operations, modular arithmetic operations or digital algebraic operations, which greatly impact the security, since attacks on these algorithms have been discovered [4-9]. For instance, X.Y. Wang found an effective method to reduce the complexity of collisions of SHA-1, issued as a Federal Information Processing Standard by NIST [5]. Chaos has some inherent merits of one way, sensitivity to tiny modifications in initial conditions and parameters, mixing property and ergodicity, which can be used for designing chaotic hash functions. K.W. Wong is the first to propose the chaotic hash function, which is built on the number of iterations of one-dimensional logistic map needed to reach the region corresponding to the character, along with a lookup table updated dynamically [10]. After then, chaotic hash functions are gradually attracting more and more researchers to study ranging from the use of simple maps, such as tent map [11-13] and logistic map [14,15], to the use of more complicated maps of the sine map [16], standard map [17], piecewise linear or nonlinear chaotic maps [18-21], and high-dimensional chaotic maps [22-24].

Since these chaotic maps in cryptanalytic studies reveal security weakness [25-32], we propose a circular shift based chaotic hash algorithm with variable parameters in this paper. We exploit piecewise linear chaotic map and one-way coupled map lattice to produce initial values and variable parameters. Circular shifts are introduced to improve the randomness of hash values. We evaluate the proposed hash algorithm in terms of distribution of the hash value, sensitivity of the hash value 
to slight modifications of the original message and secret keys, confusion and diffusion properties, robustness against birthday and meet-in-the-middle attacks, collision tests, analysis of speed, randomness tests, flexibility, computational complexity, and the results demonstrate that the proposed algorithm has strong security strength. Compared with the existing chaotic hash algorithms, our algorithm shows better statistical performance and strong collision resistance.

The remainder of this paper is organized as follows: Section 2 briefly introduces the preliminaries of piecewise linear chaotic map and one-way coupled map lattice used in our algorithm. In Section 3, we design the circular shift based chaotic hash function with variable parameters in detail, which is composed of parameter initialization, message processing and hash value generation. We excessively evaluate the performance of the proposed hash algorithm in Section 4 and present conclusions in Section 5.

\section{Preliminaries}

In this section, we briefly depict the one-dimensional piecewise linear chaotic map and the four-dimensional one-way coupled map lattice used in our hash algorithm, respectively.

\subsection{Piecewise linear chaotic map (PWLCM)}

We select the one-dimensional piecewise linear chaotic map in the proposed hash algorithm, which is expressed as: 


$$
x_{i+1}=\operatorname{PWLCM}\left(u, x_{i}\right)= \begin{cases}x_{i} / u & 0 \leq x_{i}<u \\ \left(x_{i}-u\right) /(0.5-u) & u \leq x_{i}<0.5 \\ \left(1-u-x_{i}\right) /(0.5-u) & 0.5 \leq x_{i}<1-u \\ \left(1-x_{i}\right) / u & 1-u \leq x_{i} \leq 1\end{cases}
$$

where $x_{i}$ represents the iteration trajectory value, and $u$ denotes the control parameter. When $u$ is assigned values in $(0,0.5), x_{i}$ evolves into a chaotic state in range of $(0,1)$. The PWLCM has properties of uniform distribution, good ergodicity, confusion and diffusion, therefore, it can provide chaotic random sequences. To show the quality of PWLCM, we plot the Lyapunov exponents and the bifurcation diagram for different values of control parameter $u$ in Figs. 1 and 2. As illustrated in Figs. 1 and 2, for $0<u<0.5$, PWLCM can exhibit chaotic behavior.

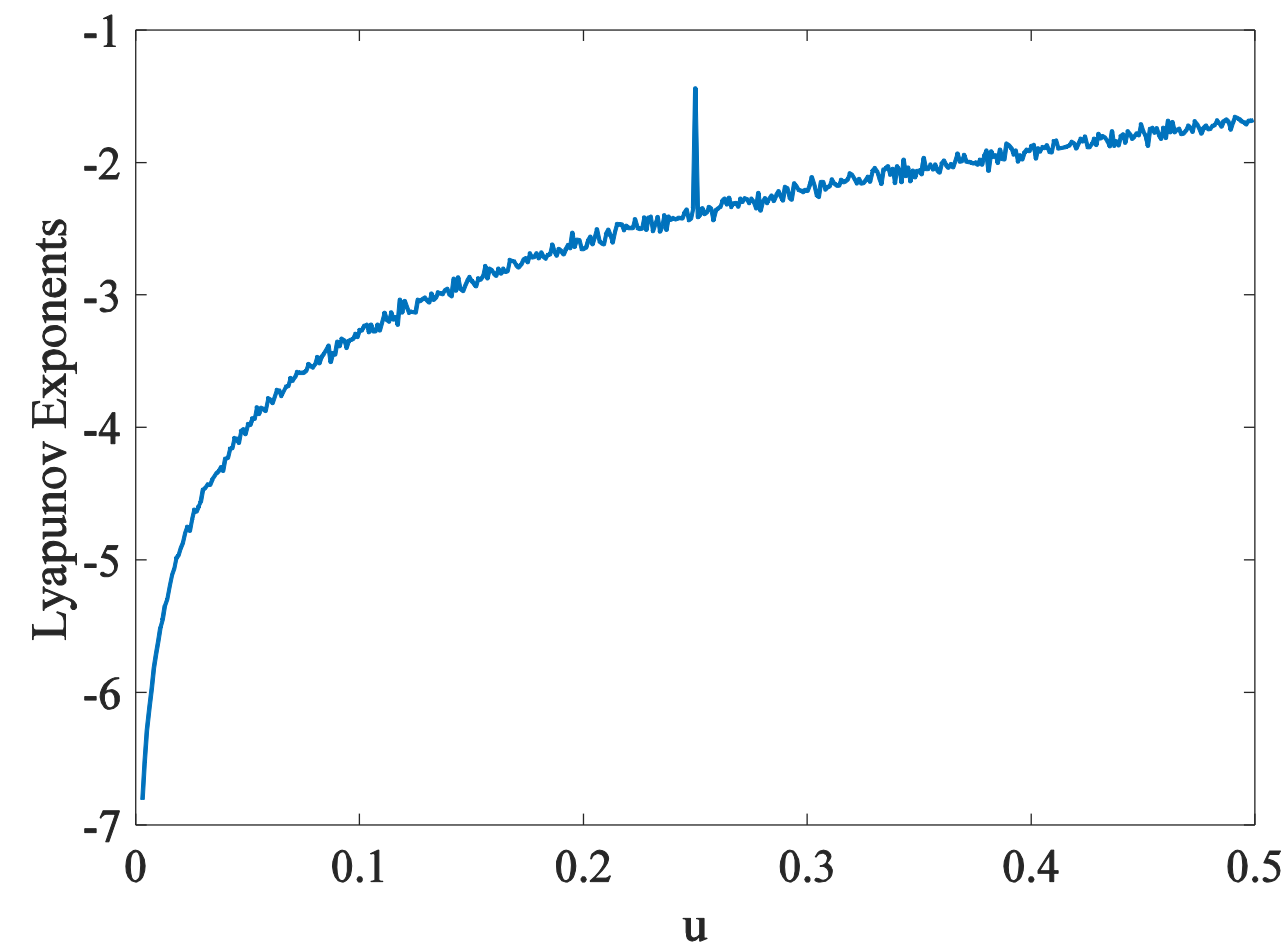

Fig.1. Lyapunov exponents of PWLCM for different control parameter $u(u \in(0,0.5))$ 


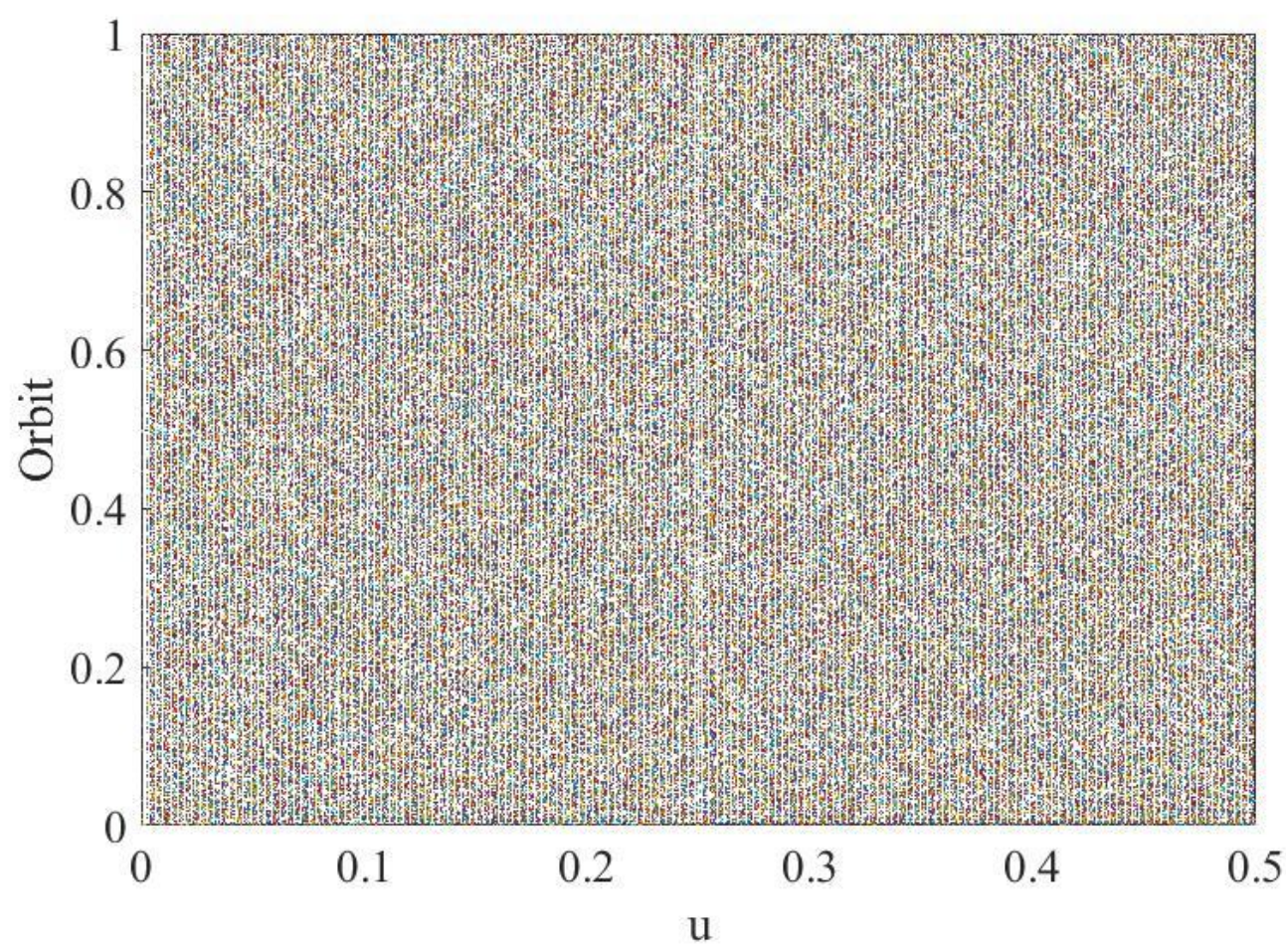

Fig.2. Bifurcation diagram of PWLCM for different control parameter $u(u \in(0,0.5))$

\subsection{One-way coupled map lattice (OCML)}

We select the four-dimensional one-way coupled map lattice in the proposed hash algorithm, which is expressed as:

$$
\left[\begin{array}{l}
x_{1}(i+1) \\
x_{2}(i+1) \\
x_{3}(i+1) \\
x_{4}(i+1)
\end{array}\right]=O C M L\left(e, x_{1}(i), x_{2}(i), x_{3}(i), x_{4}(i)\right)=\left\{\begin{array}{l}
x_{1}(i+1)=(1-e) g\left(x_{1}(i)\right)+e g\left(x_{4}(i)\right) \\
x_{2}(i+1)=(1-e) g\left(x_{2}(i)\right)+e g\left(x_{1}(i)\right) \\
x_{3}(i+1)=(1-e) g\left(x_{3}(i)\right)+e g\left(x_{2}(i)\right) \\
x_{4}(i+1)=(1-e) g\left(x_{4}(i)\right)+e g\left(x_{3}(i)\right)
\end{array}\right.
$$

where $g(x)=4 x(1-x)$, and $e$ denotes a coupling constant in the range of $(0,1)$. The four inputs $x_{1}(i), x_{2}(i), x_{3}(i)$, and $x_{4}(i)$ are in the range of $[0,1]$, and the corresponding four outputs $x_{1}(i+1), x_{2}(i+1), x_{3}(i+1)$, and $x_{4}(i+1)$ belong to the range of [0,1] as well [34]. 


\section{Description of hash algorithm}

In this section, we design the circular shift based chaotic hash function with variable parameters, which is composed of parameter initialization, message processing and hash value generation. In addition, we describe the proposed algorithm in Algorithm 1 and illustrate the structure of the proposed hash function in Figs. 3 and 4. As designed, the input is an arbitrary length of message $M^{\prime}$ and the output is $h=128$ bits of hash value.

\subsection{Parameter Initialization}

We first convert the original message $M^{\prime}$ into ASCII code values based on the ASCII code chart and then save them in an array $M$, where the length of $M$ is denoted as $l$. Then, with initial value $x_{0}=0.8$ and parameter $u_{0}=0.232323$, we iterate the PWLCM $(128+l)$ times to obtain an array $X$. Then we round the first 128 elements of $X$ to the nearest integers, and assign them to the initial hash value $H_{0}$ $\left(H_{0}=(X[1], X[2], \ldots, X[128])\right)$, where $H(i)(i=1,2, \ldots, 128)$ are binary values. Finally, we assign the rest elements of the array $X$ to a new array $U$ $(U=(X[129], X[130], \ldots, X[128+l]))$, where $U(i)(i=1,2, \ldots, l)$ are pure decimals.

\subsection{Message processing}

Since each element (character) of array $M$ is processed in the same mode, we choose $M(n)(n=1,2, \ldots, l)$ as a representative to illustrate the generation process of the $n$th middle hash value $H_{n}$. For element $M(n)$, we iterate PWLCM $i=132$ times to generate an array $u X$ with the initial value $u x_{0}=\frac{M(n)}{256}$, and variable 
parameters

$$
u u_{n}=\frac{u u_{0}+u x_{n-1}}{3}(i=1,2, \ldots, 132)
$$

where $u u_{0}=|w-\lfloor w\rfloor-0.5|$ and $w=\frac{(n+1) \times(M(n)+2)}{(n+2) \times(M(n)+1)}+U(n)$. Then, we round the first 128 elements of $u X$ into the nearest integers, and assign them to the four initial buffers $A, B, C, D$ as: $A=(u X(1), u X(2), \ldots, u X(32))$,

$$
\begin{aligned}
& B=(u X(33), u X(34), \ldots, u X(64)), \\
& C=(u X(65), u X(66), \ldots, u X(96)), \\
& D=(u X(97), u X(98), \ldots, u X(128)) .
\end{aligned}
$$

We assign the rest four elements of $u X$ as: $x_{1}=u X(129), x_{2}=u X(130), x_{3}=u X(131)$, and $x_{4}=u X(132)$, which are the input parameters of OCML. With above parameters, we iterate $\operatorname{OCML}\left(u X(i), x_{1}, x_{2}, x_{3}, x_{4}\right) \quad i=128$ times and we obtain four sequence values of $x_{1}, x_{2}, x_{3}, x_{4}$, each with length of 128 . Then, we assign values as: $\quad c h_{1}(i)=\bmod \left(\left\lfloor x_{1}(i)\right\rfloor \times 1000,32\right)+1 \quad ; \quad c l_{1}(i)=\bmod \left(\left\lfloor x_{1}(i)\right\rfloor \times 10000,32\right) \quad$; $c h_{2}(i)=\bmod \left(\left\lfloor x_{2}(32+i)\right\rfloor \times 1000,32\right)+1 \quad ; \quad c l_{2}(i)=\bmod \left(\left\lfloor x_{2}(32+i)\right\rfloor \times 10000,32\right) \quad ;$ $c h_{3}(i)=\bmod \left(\left\lfloor x_{3}(64+i)\right\rfloor \times 1000,32\right)+1 \quad ; \quad c l_{3}(i)=\bmod \left(\left\lfloor x_{3}(64+i)\right\rfloor \times 10000,32\right) \quad ;$ $c h_{4}(i)=\bmod \left(\left\lfloor x_{4}(96+i)\right\rfloor \times 1000,32\right)+1 ; c l_{4}(i)=\bmod \left(\left\lfloor x_{4}(96+i)\right\rfloor \times 10000,32\right)$.

For buffers $A, B, C$, and $D$, we conduct $i$-time $(i=1,2, \ldots, 32)$ value switching and left circular shifts as: swap $A(i)$ with $A\left(c h_{1}(i)\right)$, and then apply $c l_{1}(i)$-bit left circular shifts; swap $B(i)$ with $B\left(c h_{2}(i)\right)$, and then apply $c l_{2}(i)$-bit left circular shifts; swap $C(i)$ with $C\left(c h_{3}(i)\right)$, and then apply $c l_{3}(i)$-bit left circular shifts; swap $D(i)$ with $D\left(c h_{4}(i)\right)$, and then apply $c l_{4}(i)$-bit left circular shifts. Then, we obtain updated buffer values $A, B, C$, and $D$, which are then cascaded to generate the middle hash value $H_{n}$. Meanwhile, we 
store

values

as:

$$
\operatorname{chf}_{1}(n)=\operatorname{ch}_{1}(1)+\operatorname{ch}_{2}(1)+\operatorname{ch}_{3}(1)+\operatorname{ch}_{4}(1)
$$

$c h f_{2}(n)=c h_{1}(32)+c h_{2}(32)+c h_{3}(32)+c h_{4}(32)$, and $c l f(n)=c l_{1}(32)+c l_{2}(32)+c l_{3}(32)+c l_{4}(32)$.

\section{3 Have value generation}

For current array $M(n) \quad(n=1,2, \ldots, l), \quad H_{n}=H_{n} \oplus H_{n-1} \quad$, we swap $H_{n}\left(\operatorname{chf} f_{1}(n)\right)$ with $H_{n}\left(\operatorname{chf} f_{2}(n)\right)$, and then apply $\operatorname{clf}(i)$-bit left circular shifts. After all the messages are processed, we obtain the final hash value $H_{l}$.

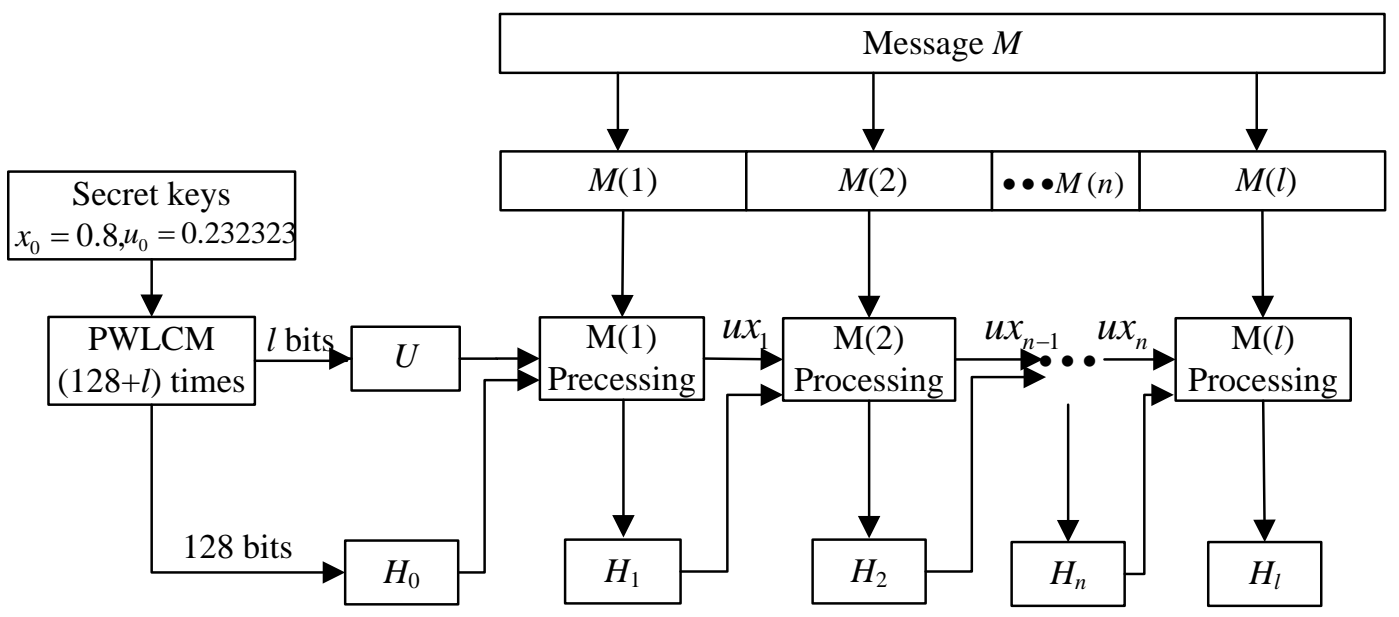

Fig.3. Structure of the proposed chaotic hash function

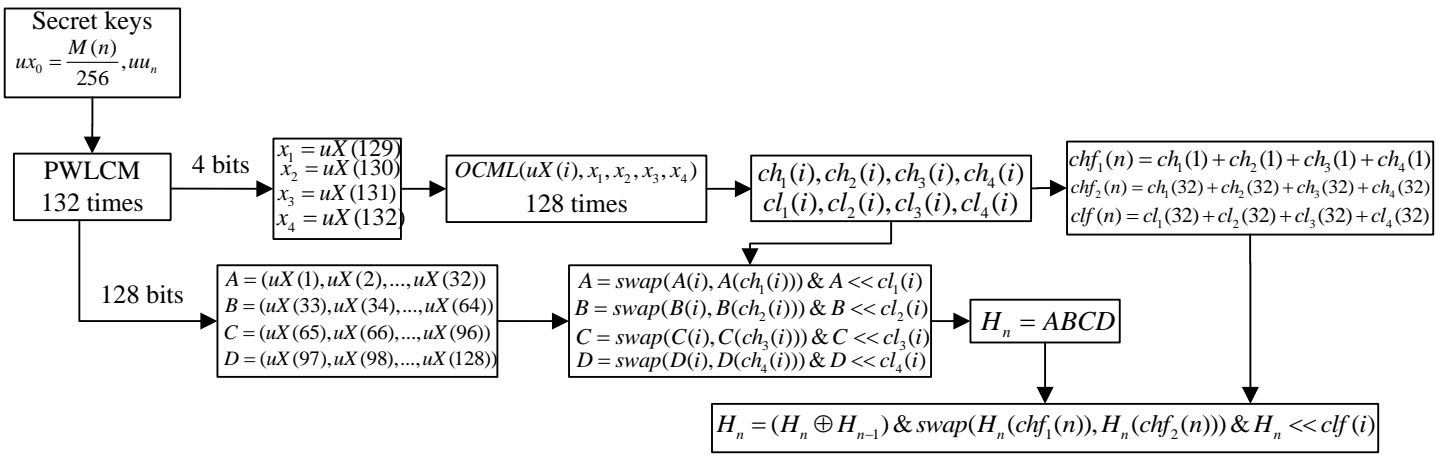

Fig.4. Processing details of message $\mathrm{M}(n)$

Algorithm 1 The circular shift based hash function construction 
Input: Secret keys $\left(x_{0}, u_{0}\right)$

Output: 128 -bit hash value $H_{l}$

1: Initialize $H_{0}\left(H_{0}=(X[1], X[2], \ldots, X[128])\right)$ and $U(U=(X[129], X[130], \ldots, X[128+l]))$ by iterating

PWLCM $(128+l)$ times with secret keys $\left(x_{0}, u_{0}\right)$

2: for $n=1$ to $l$ do

\section{// Parameter initialization}

//Message processing

3: $\quad$ Generate array $u X$ by iterating PWLCM $i(i=132)$ times with initial value $u x_{0}=\frac{M(n)}{256}$ and variable parameters $u u_{n}=\frac{u u_{0}+u x_{n-1}}{3}$, where $u u_{0}=|w-\lfloor w\rfloor-0.5|$ and $w=\frac{(n+1) \times(M(n)+2)}{(n+2) \times(M(n)+1)}+U(n)$;

4: $\quad A=(u X(1), u X(2), \ldots, u X(32))$;

5: $\quad B=(u X(33), u X(34), \ldots, u X(64))$;

6: $\quad C=(u X(65), u X(66), \ldots, u X(96))$

7: $\quad D=(u X(97), u X(98), \ldots, u X(128))$;

8: $\quad$ Generate new $x_{1}, x_{2}, x_{3}, x_{4}$ by iterating $\operatorname{OCML}\left(u X(i), x_{1}, x_{2}, x_{3}, x_{4}\right) \quad i(i=128)$ times with inputs of $x_{1}=u X(129), x_{2}=u X(130), x_{3}=u X(131), x_{4}=u X(132)$;

9: $\quad c h_{1}(i)=\bmod \left(\left\lfloor x_{1}(i)\right\rfloor \times 1000,32\right)+1 ; c l_{1}(i)=\bmod \left(\left\lfloor x_{1}(i)\right\rfloor \times 10000,32\right)$

10: $\quad c h_{2}(i)=\bmod \left(\left\lfloor x_{2}(32+i)\right\rfloor \times 1000,32\right)+1 ; c l_{2}(i)=\bmod \left(\left\lfloor x_{2}(32+i)\right\rfloor \times 10000,32\right)$;

11: $\quad c_{3}(i)=\bmod \left(\left\lfloor x_{3}(64+i)\right\rfloor \times 1000,32\right)+1 ; c l_{3}(i)=\bmod \left(\left\lfloor x_{3}(64+i)\right\rfloor \times 10000,32\right)$

12: $\quad c h_{4}(i)=\bmod \left(\left\lfloor x_{4}(96+i)\right\rfloor \times 1000,32\right)+1 ; c l_{4}(i)=\bmod \left(\left\lfloor x_{4}(96+i)\right\rfloor \times 10000,32\right)$;

11: $\quad$ for $i=1$ to 32 do

12: $\quad$ swap $A(i)$ with $A\left(c h_{1}(i)\right)$, and apply $c l_{1}(i)$-bit left circular shifts;

13: $\quad$ swap $B(i)$ with $B\left(c h_{2}(i)\right)$, and apply $c l_{2}(i)$-bit left circular shifts;

14: $\quad$ swap $C(i)$ with $C\left(\operatorname{ch}_{3}(i)\right)$, and apply $c l_{3}(i)$-bit left circular shifts;

15: $\quad$ swap $D(i)$ with $D\left(c_{4}(i)\right)$, and apply $c l_{4}(i)$-bit left circular shifts; 
16: end for

17: $\quad H_{n}=$ cascaded $A, B, C, D ; \quad H_{n}=H_{n} \oplus H_{n-1} ; \quad$ //Middle hash value generation

18: $\quad \operatorname{ch} f_{1}(n)=c h_{1}(1)+c h_{2}(1)+c h_{3}(1)+c h_{4}(1)$

19: $\quad \operatorname{chf}_{2}(n)=\operatorname{ch}_{1}(32)+\operatorname{ch}_{2}(32)+\operatorname{ch}_{3}(32)+\operatorname{ch}_{4}(32)$

20: $\quad c l f(n)=c l_{1}(32)+c l_{2}(32)+c l_{3}(32)+c l_{4}(32)$

21: $\quad \operatorname{swap} H_{n}\left(c h f_{1}(n)\right)$ with $H_{n}\left(\operatorname{chf} f_{2}(n)\right)$, and apply $\operatorname{clf}(i)$-bit left circular shifts;

22: end for

23: Return $H_{l}$

//Hash value generation

\section{Performance analysis}

We evaluate the performance of the circular shift based chaotic hash function in terms of distribution of the hash value, sensitivity of the hash value to slight modifications of the original message and secret keys, confusion and diffusion properties, robustness against birthday and meet-in-the-middle attacks, collision tests, analysis of speed, randomness tests, flexibility, computational complexity, and comparison with other algorithms. To evaluate the performance of the proposed hash algorithm, we randomly choose an arbitrary of length of message as:

"Southwest University (SWU) is a key comprehensive university, under the direct administration of the Ministry of Education. It was newly established in July 2005 through the incorporation of former Southwest China Normal University and Southwest Agricultural University upon the approval of the Ministry of Education. SWU is situated nearby the beautiful Jialing River, and 
is located at the foot of Jinyun Mountain, a state level scenic spot, in Beibei

District, Chongqing Municipality.”

\subsection{Distribution of the hash value}

The characteristic of uniform distribution of hash value directly relates to the security of a hash function. With the randomly chosen message, we simulate the proposed hash algorithm to generate the hash value, and the distribution of both the message and the corresponding hash value are plotted. As depicted in Fig. 5(a), the random chosen message spreads in a small area. This is because the ASCII code values of printable characters (message) in ASCII code chart locate in the range of [32, 126]. Nevertheless, the hexadecimal hash value spreads around randomly, as shown in Fig. 5(b). In contrast, we perform the same experiment on a particular "blank space" message with same length, and the distribution of the particular message and the hash value are plotted as well. As illustrated in Fig. 6, even under this very extreme condition, the distribution of hash value is uniform as well. Therefore, the hash value of the proposed hash algorithm has a good characteristic of uniform distribution. 

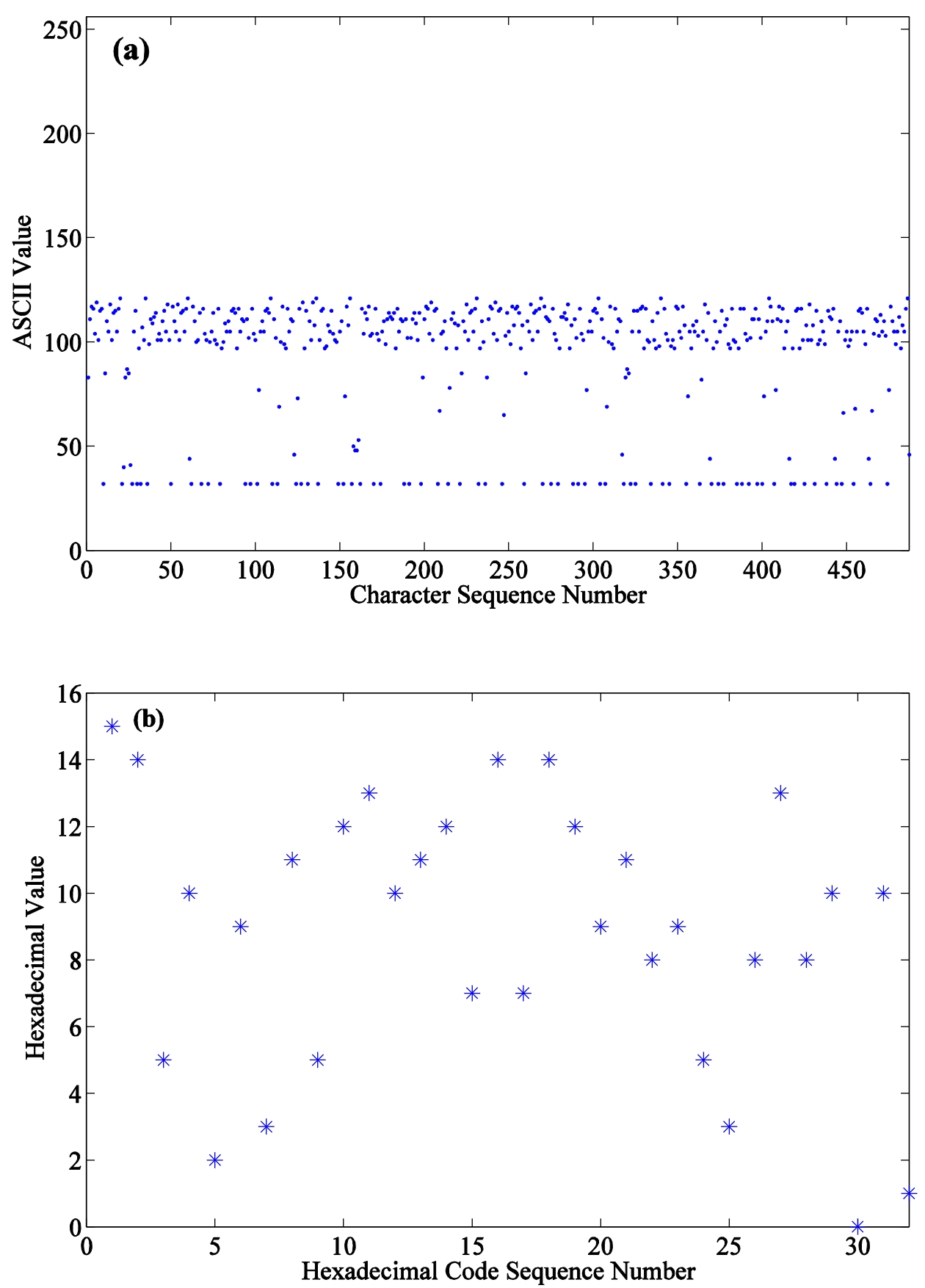

Fig.5. Distribution of randomly chosen message and hash value: (a) distribution of the message in ASCII code; (b) distribution of the hexadecimal hash value (FE5A293B5CDABC7E7EC9B89538D8A0A1) 

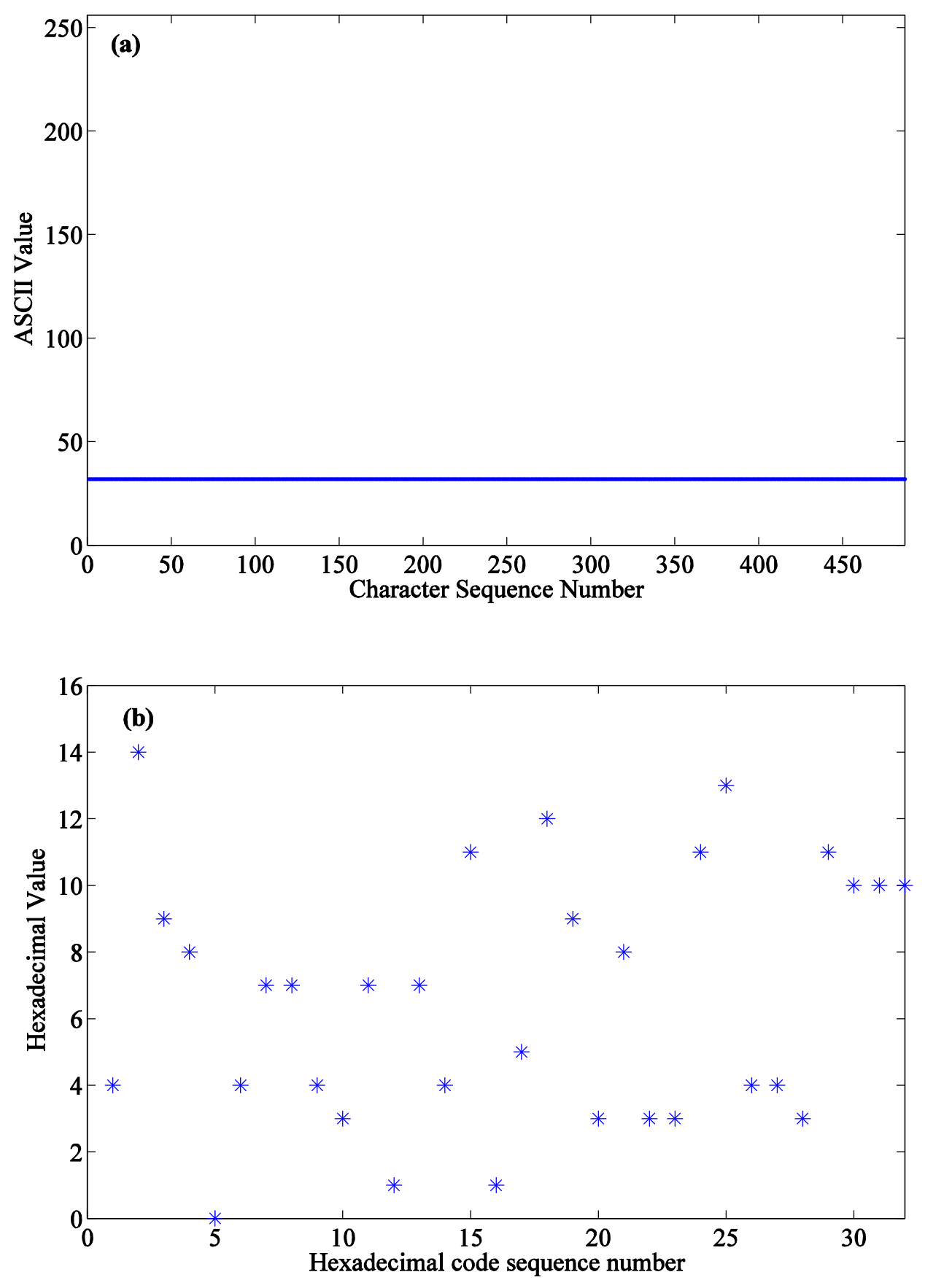

Fig.6. Distribution of the particular "blank space" message and hash value: (a) distribution of the "blank space" message; (b) distribution of the hexadecimal hash value (4E980477437174B15C93833BD443BAAA)

\subsection{Sensitivity to the original message and secret key}


Hash function is a many-to-one map since its domain is much larger than its range. According to the characteristic of a hash function, it is hard to find the original message if the hash value is known, which indicates that a hash function must be sensitive to tiny modifications in original message or secret key. In particular, any slight modifications on message or secret key will lead to a $50 \%$ difference in hash value, according to a Hamming distance of approximately $n / 2$ ( $n$ denotes the length of hash value) between the two hash values. We simulate the hash algorithm under eight different conditions to evaluate the sensitivity of tiny modifications to the original message and secret key.

$\mathrm{C} 1$ : The original message is the randomly chosen message;

C2: Modify the first character " $S$ " in original message to " $s$ ";

C3: Add a number " 1 " in front of the original message;

C4: Modify "July" in the middle of the message to "June";

C5: Swap the "China Normal University" and "Southwest Agricultural University" in the original message;

C6: Modify the full stop "." at the end of original message to a comma ",";

C7: Add a "blank space" at the end of the original message;

C8: Modify the secret key $x_{0}=0.232323$ to $x_{0}=0.232323000000001$.

We list the corresponding hash values in hexadecimal formats associated with the eight conditions, followed by the bit differences of hash values comparing with Condition 1 in the parenthesis:

C1: FE5A293B5CDABC7E7EC9B89538D8A0A1 
C2: DBC3A7DCBEDE048F4F35AFDD0B7EFEF8 (63)

C3: B1C87925F2448D08B7A8270C661285DB (66)

C4: 9BDA624D5463F2F4CB6BAC13C05A9E10 (56)

C5: 7B3366ADDE7E280EDCB92C7171655BB7 (59)

C6: F8055075C0437A6E7BDC4ED72DD3620E (58)

C7: 7C8CD1DA337133AB6D17814EB03C8D6D (70)

C8: 7B23D05E1C84CDC48FDF800852FBBFA7 (63)
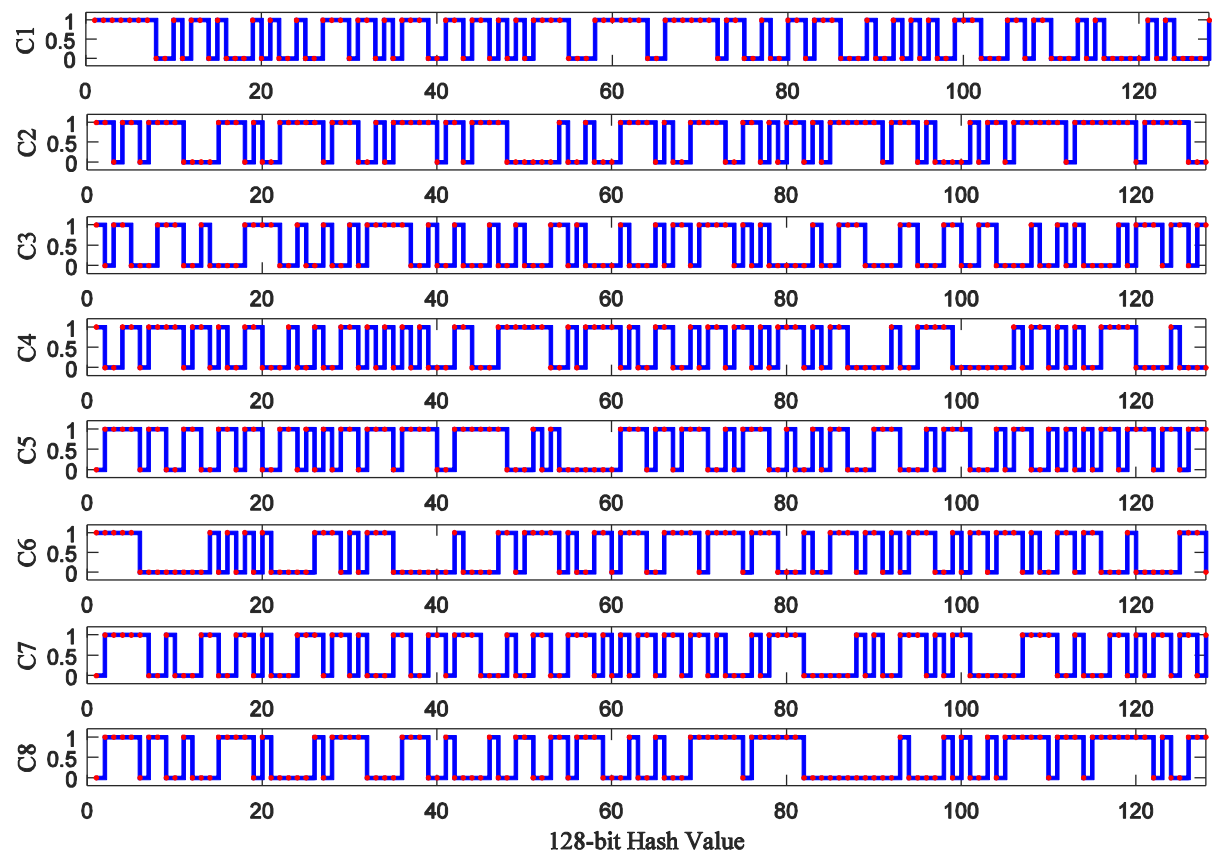

Fig.7. Hash values under eight different conditions

We illustrate the corresponding binary sequences of hash values in Fig.7. As depicted in Fig.7, the sensitivity property of the hash algorithm is so good that any tiny modification of the message ( $\mathrm{C} 1$ to $\mathrm{C} 7)$ causes huge difference in the hash value. In addition, a tiny change on the key (C8) leads to 63-bit difference, which is close to 
the ideal value of 64 . Based on the experiment, the sensitive precision of secret key $x_{0}$ to hash value is $10^{-16}$, and the sensitive precision of the parameter $u$ is $10^{-16}$ as well. Considering both $x_{0} \in(0,1)$ and $u \in(0,0.5)$, we can derive that the secret key space is approximately larger than $2^{106}$.

\subsection{Statistical analysis of confusion and diffusion}

Shannon introduces confusion and diffusion as two of the key features of a secure cipher, which are regarded as basic design criteria for any cryptographic algorithm [35]. In cryptography, confusion refers to as the relationship between a message and its corresponding hash value must be complex and unpredictable, while diffusion refers to as the hash value is highly dependent on the message. In particular, with good diffusion, a 1-bit modification in a message leads to a significant change in the hash value.

In order to capture qualitative characteristics of the diffusion and confusion capabilities of the proposed hash algorithm, we conduct the following experiment: first, we obtain the hash value of a randomly chosen message. Then, we randomly modify a bit in the original message, and generate the corresponding hash value. Finally, we compare the two hash values and count the number of different bits at the same position in the hash values denoted as $B_{i}$. Besides, in order to capture the statistic characteristics of diffusion and confusion, we introduce six statistical measures:

Minimum changed bit number: $B_{\min }=\min \left\{B_{1}, B_{2}, \ldots, B_{\mathrm{N}}\right\}$ Maximum changed bit number: $B_{\max }=\max \left\{B_{1}, B_{2}, \ldots, B_{\mathrm{N}}\right\}$, 
Mean changed bit number: $\bar{B}=\frac{1}{N} \sum_{i=1}^{N} B_{i}$,

Mean changed probability: $P=(\bar{B} / h) \times 100 \%$,

Standard variance of the changed bit number: $\Delta B=\sqrt{\frac{1}{N-1} \sum_{i=1}^{N}\left(B_{i}-\bar{B}\right)^{2}}$,

Standard variance: $\Delta P=\sqrt{\frac{1}{N-1} \sum_{i=1}^{N}\left(B_{i} / h-P\right)^{2}} \times 100 \%$, where $N$ indicates the test time of experiments, and $h$ denotes the length of hash value.

The experiment is performed $N$ times on our hash algorithm, where $N=256,512$, 1024,2048 , and 10000. The corresponding results of $B_{\min }, B_{\max }, \bar{B}, P, \Delta B$, and $\Delta P$ are tabulated in Table 1. The corresponding distribution of changed bit number $\bar{B}$, is illustrated in Fig. 8.

Table 1 Statistics of number of changed bits

\begin{tabular}{lllllll}
\hline$N$ & $N=256$ & $N=512$ & $N=1024$ & $N=2048$ & $N=10000$ & Mean \\
\hline$B_{\min }$ & 48 & 47 & 47 & 47 & 47 & 47.2 \\
$B_{\max }$ & 77 & 81 & 79 & 81 & 81 & 79.8 \\
$\bar{B}$ & 63.8627 & 64.0783 & 64.0479 & 64.1700 & 64.0415 & 64.0401 \\
$P(\%)$ & 49.89 & 50.06 & 50.04 & 50.13 & 50.03 & 50.03 \\
$\Delta B$ & 5.4974 & 5.7628 & 5.8880 & 5.7488 & 5.7929 & 5.7380 \\
$\Delta P(\%)$ & 4.29 & 4.50 & 4.60 & 4.49 & 4.53 & 4.48 \\
\hline
\end{tabular}



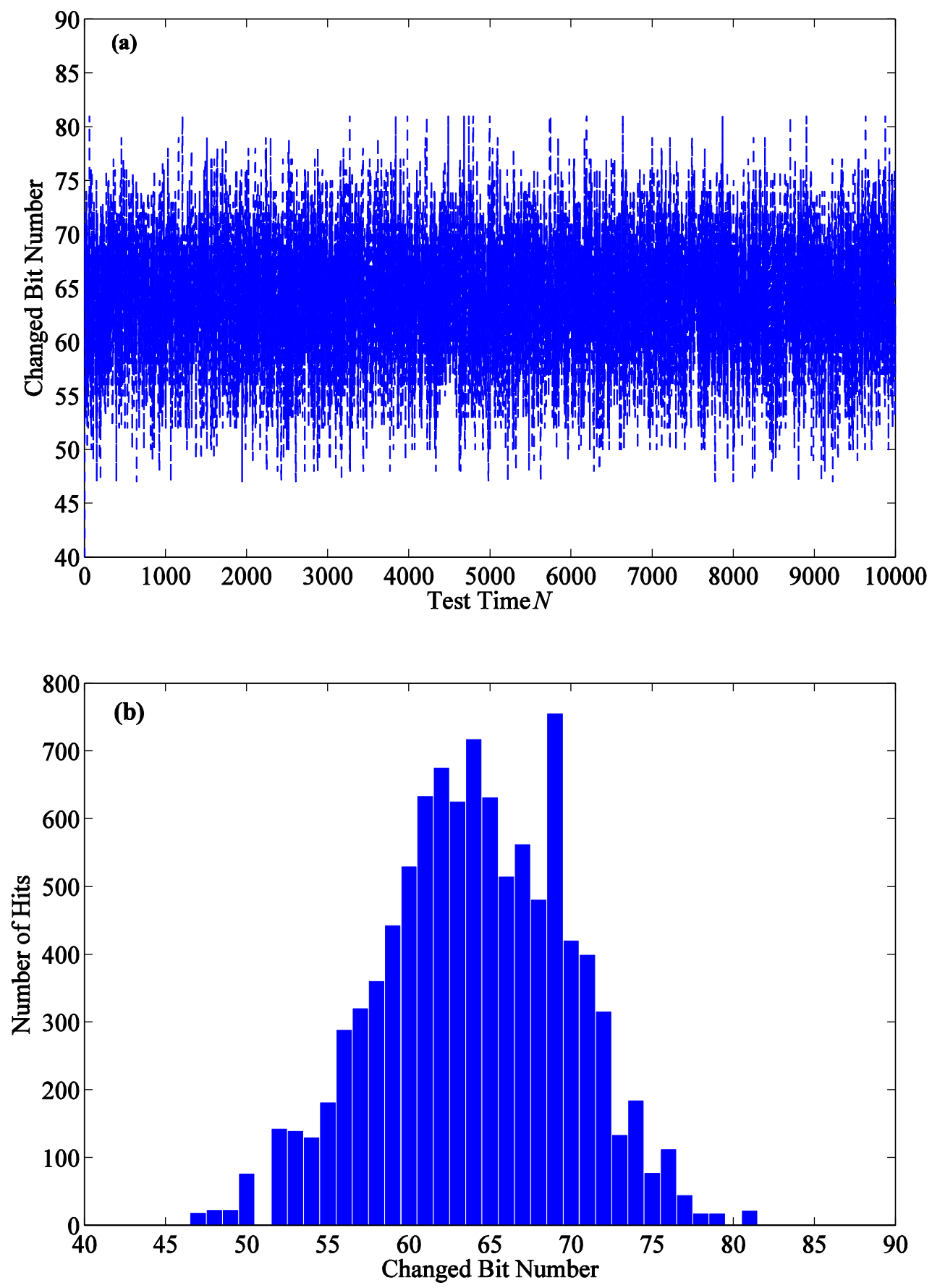

Fig.8. Spread of changed bit number: (a) Plot of $B_{i}$, (b) Histogram of $B_{i}$.

Based on the statistical experiment results, the proposed hash function has a mean changed bit number $\bar{B}=64.0401$ and mean changed probability $P=50.03$ that are very close to the ideal values of 64 bits and $50 \%$, respectively. The values of the 
standard variances are also very small, which indicate a strong capability for confusion and diffusion. Besides that, as illustrated in Fig. 8(a), the plot of $B_{i}$ shows that its value is evenly distributed, and as shown in Fig. 8(b), the histogram of $B_{i}$ has a normal distribution centering on the ideal value of 64 . These results indicate that the proposed hash function has near-ideal statistical qualities in terms of confusion and diffusion strength, whereby any change of even 1 bit of the plaintext will lead to a completely different message digest.

\subsection{Collision analysis}

A collision refers to as two distinct messages produce the same hash value, while collision attack indicates that it tries to find two arbitrary messages that collide. Collision resistance is a property of a hash function: a hash function $h$ is collision resistant if it is hard to find any two distinct messages $M$ and $M^{\prime}$ that hash to the same output, such that $h(M)=h\left(M^{\prime}\right)$. In this section, we first perform qualitative analysis on birthday and meet-in-the-middle attacks and then conduct quantitative analysis on collision resistance.

\subsubsection{Birthday and meet-in-the-middle attacks}

Birthday attack is an algorithm-independent attack, where a set of random messages are inspected for a possible pair of messages whose hash values collide $[49,50]$. For an $h$-bit hash function, the complexity of this attack is about $2^{\frac{h}{2}}$. Since the length of hash value of the proposed algorithm $h \geq 128$, birthday attacks are rendered impractical. 
Meet-in-the-middle attack is another generic attack applied to cryptographic systems composed of different layers. The attack is to find collisions on intermediate hash results rather than the final hash value. For example, assuming that the original message is described as $M=\left(M_{1}, M_{2}, \ldots, M_{l-1}, M_{l}\right)$, then the expected contradicted message can be represented as $M^{\prime}=\left(M_{1}, M_{2}, \cdots, M_{l-1}, M_{l}^{\prime}\right)$. As we tested in Section 4.2, any character modification will cause huge difference in the final hash value. Therefore, the proposed hash algorithm is robust against meet-in-the-middle attacks.

\subsubsection{Collision tests}

In order to show quantitative analysis on collision resistance, we conduct the experiment: first, we generate the hash value of a randomly chosen message and then store it in ASCII code format. Then, we randomly modify a bit in the message, obtain a new hash value, and store it in the same format as well. Finally, we compare the two produced hash values by counting the number of the same ASCII character at the same location, that is, the number of hits. We conducted $N=10000$ times of the experiment, and plot the distribution of the number of hits in Fig.9. As depicted in Fig.9 and Table 2, 505 tests hit once, while in 9495 tests, no hit occurs. The maximum number of equal characters is only 1 , therefore, the collision on our hash algorithm is very low. 


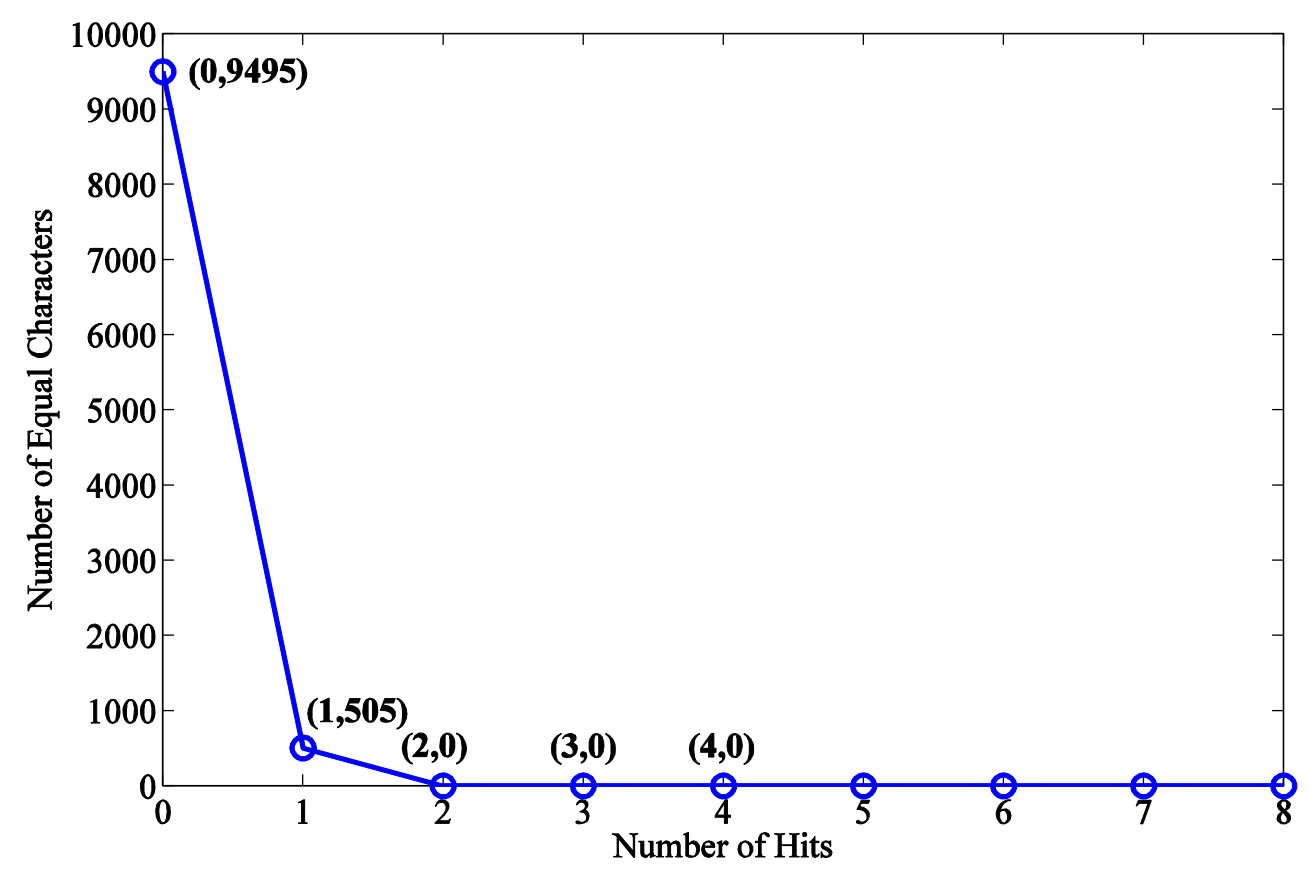

Fig.9. Distribution of the number of the same ASCII characters at the same location in hash value

Table 2 Number of hits

\begin{tabular}{llllllllll}
\hline Number of equal characters & 0 & 1 & 2 & 3 & 4 & 5 & 6 & 7 & 8 \\
\hline The proposed algorithm & 9495 & 505 & 0 & 0 & 0 & 0 & 0 & 0 & 0 \\
\hline
\end{tabular}

In addition, we compare the hash values by sequences of bytes, which can be represented by an ASCII character. We denote the decimal value of an ASCII character $e$ by $t(e)$. For two messages $M$ and $M^{\prime}$, we calculate the hash values $h$ and $h^{\prime}$. Let $e_{i}$ denote the $i$ th ASCII character of hash value $h$ while $e_{i}^{\prime}$ represents the $i$ th ASCII character of hash value $h^{\prime}$. Then, we compute the absolute difference between the two hash values $h$ and $h^{\prime}$ by the equation: $d=\sum_{i=1}^{N}\left|t\left(e_{i}\right)-t\left(e_{i}^{\prime}\right)\right|$. We perform the collision test $N=10000$ times, and the corresponding results of the maximum, minimum, mean and 
mean/character values of $d$ are listed in Table 3. As described in Table 3, the mean/character of absolute difference $d$ of two hash values is 78.31. For the absolute difference $d$, the theoretical mean/character value computed in $[36,48,50,58]$ is 83.3333 , therefore, our mean/character value is close to the theoretical value and the analysis on collision shows that our hash algorithm has strong collision resistance. Moreover, as described in $[18,58,59]$, the ideal value of sum of absolute difference for two uniform distributions is $2 / 3$ of the mean value for the distribution. The ideal mean value of the absolute difference for 128-bit hash value is equal to 1360 , while our mean value is 1253 .

Table 3 Absolute differences $d$ of two hash values

\begin{tabular}{llll}
\hline Maximum & Minimum & Mean & Mean/Character \\
\hline 1972 & 685 & 1253 & 78.31 \\
\hline
\end{tabular}

\subsection{Analysis of speed}

The time required to generate the hash value is directly related to the message processing procedure, according to the structure of the proposed hash algorithm. To analyze the compression speed, we implement the proposed hash algorithm in C99 on a PC with 2.50GHz Intel Pentium IV Dual-core, 2G Memory and Ubuntu 10.10 operation system and the test message is composed of 10000 ASCII codes. Moreover, we implement the well-known and widely used MD5 [36] and SHA-1 [51] algorithms in C99 with optimized codes on the same settings. Finally, we present the average compression speed comparison based on specified settings with some representative 
hash algorithms $[18,34,38,43,49,52,53]$ in Table 4. As shown in Table 4, the average compression speed of the proposed algorithm (132.07 Mbps) is higher than SHA-1 (114.45 Mbps) and is very close to MD5 (132.10 Mbps). In addition, the algorithm of [18] shows higher compression speed, and Yi's algorithm [52] illustrates interesting compression speed, since the settings of the implementation are far behind ours.

Table 4 Average compression speed comparison

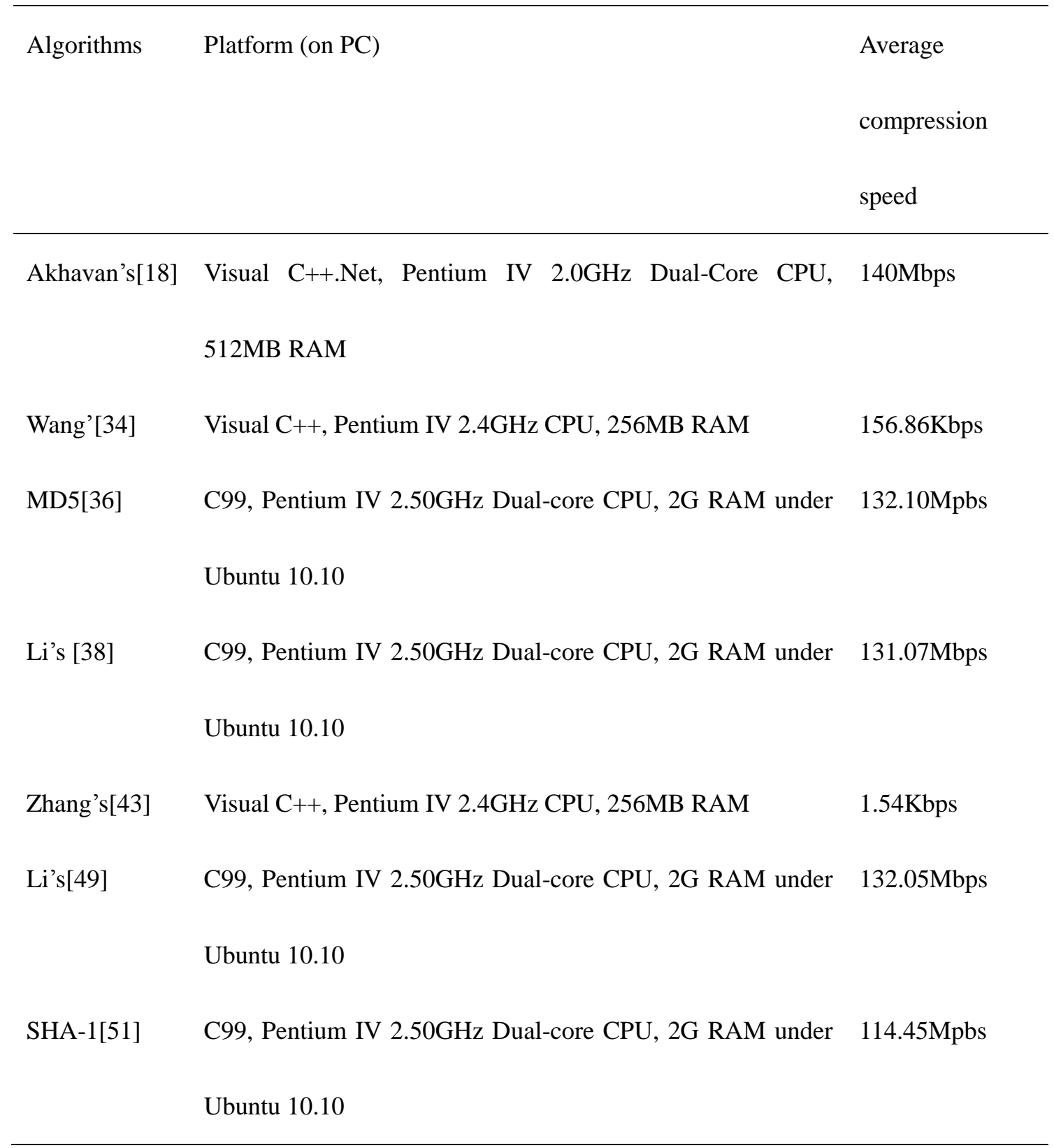



Yi's[52]
A software on Pentium III 667MHz CPU under Win98
$8.42 \mathrm{Mbps}$
Guo's[53]
C99, Pentium IV 2.50GHz Dual-core CPU, 2G RAM under
$131.25 \mathrm{Mbps}$

Ubuntu 10.10

This algorithm

C99, Pentium IV 2.50GHz Dual-core CPU, 2G RAM under

132.07Mbps

Ubuntu 10.10

\subsection{Randomness tests}

In order to show the randomness of the proposed hash algorithm, we perform a preliminary security analysis, consisting in examining the statistical properties of its output over a low-entropy input. By randomly modifying one bit of the original message each time, we generate a sequence of hash values constructed a file of 250 MB to be analyzed with four batteries of statistical tests: NIST [54], Diehard [55], and ENT [56], and the most stringent testing TestU01 [57,58]. The results obtained with NIST, Diehard, ENT, and TestU01 are presented in Tables 5, 6, 7, and 8, respectively. In Table 5, we calculate $100 p$-values for every test in the statistical suite and present the proportion of successful ones. If this proportion is lower than 0.96 , it is considered that the whole test failed. In Table 6 , we first calculate several $p$-values in the same test, and then summarize them by a Kolmogorov-Smirnov $p$-value (marked with *), where if it is greater than 0.05 , it is considered successful. In Table 8 , we evaluate three different crush type batteries: Small Crust (which consists of 10 tests), Crust (96 tests), and Big Crust (106 tests).

From the results in Tables 5, 6, 7, and 8, we can conclude that the hash values of the proposed hash algorithm successfully pass these randomness tests, even with the 
hash values being obtained from an extremely low-entropy input.

Table 5 Proportion results obtained with the NIST suite

\begin{tabular}{llll}
\hline \multicolumn{1}{c}{ Test } & Proportion & \multicolumn{1}{c}{ Test } & Proportion \\
\hline Frequency & 0.97 & Random-excursions & $0.98,0.97$ \\
Block-frequency & 0.97 & & $0.99,0.98$ \\
Cumulative-sums & $0.97,0.97$ & & $1.00,0.99$ \\
Runs & 1.00 & & $0.98,1.00$ \\
Longest-run & 1.00 & Random-excursions-variant & $1.00,0.99,1.00$ \\
Rank & 0.98 & & $0.99,1.00,1.00$ \\
FFT & 0.99 & & $1.00,1.00,1.00$ \\
Overlapping-templates & 0.97 & & $1.00,1.00,1.00$ \\
Universal & 0.97 & & $0.99,0.99,0.98$ \\
Apen & 0.98 & & $0.97,1.00,0.98$ \\
Linear-complexity & 0.99 & & 0.99
\end{tabular}

Table 6 Test results obtained with the Diehard suite

\begin{tabular}{lllll}
\hline \multicolumn{1}{c}{ Test } & $p$-value & Test & $P$-value \\
\hline Birthday Spacings & 0.224 & Count the 1 s in a 0.665 \\
& & Stream of Bytes & \\
GCD & $0.776^{*}$ & Count the 1 is in $0.587^{*}$ \\
& & Specific Bytes
\end{tabular}




\begin{tabular}{lllll} 
Overlapping Permutations & 0.425 & Parking Lot Test & 0.446 \\
Ranks of 31x31 and 32x32 Matrices & 0.699 & Minimum & Distance & 0.433 \\
& & Test & \\
Ranks of 6x8 Matrices & 0.532 & Random Spheres Test & 0.799 \\
Monkey Tests on 20-bit Words & $0.321^{*}$ & The Squeeze Test & 0.902 \\
Monkey Test OPSO & $0.453^{*}$ & Overlapping Sums Test & 0.201 \\
Monkey Test OQSO & $0.738^{*}$ & Runs up and down Test & 0.192 \\
Monkey Test DNA & $0.688^{*}$ & The Craps Test & $0.443^{*}$ \\
\hline Overall p-value & & 0.532 &
\end{tabular}

Table 7 Test results obtained with ENT

\begin{tabular}{ll}
\hline \multicolumn{1}{c}{ Test } & \multicolumn{1}{c}{ Results } \\
\hline Entropy & 7.999996 bit/byte \\
Compression Rate & $0 \%$ \\
$\chi^{2}$ Statistic & $256.10(50 \%)$ \\
Arithmetic Mean & 127.7705 \\
Monte Carlo $\pi$ Estimation & $3.140523711(0.00 \%)$ \\
Serial Correlation Coefficient & -0.000053 \\
\hline
\end{tabular}

Table 8 Test results obtained with TestU01 test suite

\begin{tabular}{llll}
\hline Battery & Parameters & Number of statistics & Results \\
\hline Small Crush & Standard & 10 & Pass \\
\hline
\end{tabular}




$\begin{array}{llll}\text { Crush } & \text { Standard } & 96 & \text { Pass }\end{array}$

$\begin{array}{llll}\text { Big Crush } & \text { Standard } & 106 & \text { Pass }\end{array}$

\subsection{Flexibility}

To show the flexibility of the proposed hash algorithm, if we modify the viable parameter generation method of PWLCM for each message block to be a constant value, then we will obtain a parallel message processing algorithm. Since the focus of the proposed algorithm is a variable parameter for each message block which is close related to previous message block to keep message information, then we will leave the parallel solution to future work.

\subsection{Computational complexity}

The computational complexity of the proposed hash algorithm depends on the iteration operations of PWLCM and OCML and message length to be processed. First, there are about $128+l$ times of iteration of PWLCM for parameter initialization, and the time complexity is $O(n)$. Then, for each message block, the following steps will be executed $O(l)$ times: 132 times of iteration of PWLCM, 128 times of iteration of OCML, 13 times of value assignments, $O(32)$ times of value swapping and circular shifts, and then the corresponding time complexity is $O\left(n^{2}\right)$. Therefore, the total computational complexity of the proposed algorithm is $O\left(n^{2}\right)$.

\subsection{Comparison with other hash algorithms}

We perform a comparison between the proposed hash function and some significant chaos-based hash functions as well as MD5, which is based on statistical performance and collision resistance. 
Tables 9 and 10 describe the comparison of statistical performance between our algorithm and selected existing excellent algorithms. Note that the results reported in Table 9 are based on $N=2048$ random tests and 128-bit hash value, while the results of Table 10 focus on $N=10000$ random tests and 128-bit hash value. Based on the results, our algorithm shows better statistical performance.

Table 9 Comparison on statistical performance with $N=2048$ random tests

\begin{tabular}{|c|c|c|c|c|c|}
\hline \multirow[t]{2}{*}{ Schemes } & \multirow{2}{*}{$\begin{array}{l}\text { Maximum hit } \\
\text { numbers }\end{array}$} & \multicolumn{4}{|c|}{ Statistical performance of the schemes } \\
\hline & & $\bar{B}$ & $P(\%)$ & $\Delta B$ & $\Delta P(\%)$ \\
\hline MD5[36] & N/A & 64.03 & 50.02 & 5.66 & 4.42 \\
\hline Li’s[19] & 2 & 63.5684 & 49.66 & 7.4260 & 5.80 \\
\hline Xiao’s[20] & 2 & 63.9185 & 49.94 & 5.6172 & 4.39 \\
\hline Xiao's[21] & 2 & 64.0918 & 50.07 & 5.4832 & 4.28 \\
\hline Kanso’s[22] & N/A & 63.94 & 49.95 & 5.69 & 4.44 \\
\hline Deng's[25] & 2 & 63.84 & 49.88 & 5.88 & 4.59 \\
\hline Guo’s[29] & N/A & 63.399 & 49.53 & 7.127 & 6.3492 \\
\hline Wang's[34] & N/A & 63.98 & 49.98 & 5.53 & 4.33 \\
\hline Li’s[37] & 2 & 63.8076 & 49.8497 & 5.7563 & 4.4971 \\
\hline Li’s[38] & 2 & 63.89 & 49.91 & 5.64 & 4.41 \\
\hline Ren's[39] & 2 & 63.92 & 49.94 & 5.78 & 4.52 \\
\hline Xiao’s[40] & 2 & 64.0098 & 50.01 & 5.7236 & 4.47 \\
\hline Xiao's[41] & 2 & 64.1777 & 50.15 & 5.6700 & 4.405 \\
\hline
\end{tabular}




\begin{tabular}{llllll}
\hline Zhang's[42] & N/A & 63.91 & 49.92 & 5.58 & 4.36 \\
Zhang's[43] & 4 & 64.43 & 49.96 & 5.568 & 4.506 \\
Kanso's[44] & 2 & 64.01 & 50.01 & 5.61 & 4.38 \\
Teh's[45] & N/A & 64.0078 & 50.0061 & 5.6631 & 4.2558 \\
Wang's[46] & N/A & 64.153 & 50.119 & 5.767 & 4.506 \\
This paper & 1 & 64.1700 & 50.13 & 5.7488 & 4.49 \\
\hline
\end{tabular}

Table 10 Comparison on statistical performance with $N=10000$ random tests

\begin{tabular}{llllcc}
\hline \multirow{2}{*}{ Schemes } & Maximum hit & \multicolumn{4}{c}{ Statistical performance of the schemes } \\
\cline { 3 - 6 } & numbers & $\bar{B}$ & $P(\%)$ & $\Delta B$ & $\Delta P(\%)$ \\
\hline Kanso's[22] & 1 & 63.94 & 49.95 & 5.64 & 4.41 \\
Wang's[34] & 2 & 63.90 & 49.91 & 5.58 & 4.36 \\
Ren's[39] & 2 & 64.004 & 50.003 & 5.620 & 4.391 \\
Zhang's[42] & 2 & 63.96 & 49.97 & 5.52 & 4.32 \\
Teh's[45] & 1 & 63.8518 & 49.8842 & 5.6701 & 4.4298 \\
Wang's[46] & 2 & 63.986 & 49.988 & 5.616 & 4.388 \\
This paper & 1 & 64.0415 & 50.03 & 5.7929 & 4.53 \\
\hline
\end{tabular}

In addition, Tables 11 and 12 present the comparison of the number of the same ASCII characters at the same location and absolute difference $d$ in 128-bit hash values between our algorithm and selected algorithms. Note that the reported results of Table 11 are based on $N=2048$ random tests, while the results of Table 12 focus on $N=$ 
10000 random tests. Based on the results, the proposed algorithm shows better collision resistance. However, the ideal mean value of the absolute difference for 128-bit hash value is equal to $1360[18,58,59]$, while our mean value is 1255 when $N$ $=2048$ and 1253 when $N=10000$, which shows a moderate collision resistance of the proposed algorithm comparing to some algorithms.

Table 11 Comparison on collision resistance with $N=2048$ random tests

\begin{tabular}{cccccccccc}
\hline & \multicolumn{3}{c}{ Number of hits } & \multicolumn{3}{c}{ Absolute difference $d$} \\
\cline { 2 - 8 } Schemes & 0 & 1 & 2 & 3 & Min. & Max. & Mean & Mean/Char. \\
\hline Xiao's[20] & 1924 & 120 & 4 & 0 & 658 & 2156 & 1431.1 & 89.46 \\
Xiao's[21] & 1915 & 132 & 1 & 0 & 812 & 2034 & 1349.1 & 84.32 \\
Deng's[25] & 1940 & 104 & 4 & 0 & 583 & 2206 & 1399.8 & 87.49 \\
Xiao's[40] & 1926 & 120 & 2 & 0 & 605 & 1952 & 1227.8 & 76.74 \\
Xiao's[41] & 1932 & 114 & 2 & 0 & 573 & 2224 & 1401.1 & 87.56 \\
Li's[37] & 1928 & 118 & 2 & 0 & 687 & 2220 & 1432.1 & 89.51 \\
Kanso's[44] & 1954 & 92 & 2 & 0 & 731 & 2230 & 1368 & 85.5 \\
Luo's[47] & 1928 & 117 & 3 & 0 & 796 & 2418 & 1598.6 & 99.91 \\
This paper & 1945 & 103 & 0 & 0 & 685 & 1972 & 1255 & 78.44 \\
\hline
\end{tabular}

Table 12 Comparison on collision resistance with $N=10000$ random tests

\begin{tabular}{lllllllll}
\hline & \multicolumn{4}{c}{ Number of hits } & \multicolumn{3}{c}{ Absolute difference $d$} \\
\cline { 2 - 8 } Schemes & 0 & 1 & 2 & 3 & Min. & Max. Mean & Mean/Char.
\end{tabular}




\begin{tabular}{lcccccccc}
\hline Akhavan's[18] & 9434 & 553 & 13 & 0 & 744 & 2431 & 1371 & 85.69 \\
Wang's[34] & 9377 & 607 & 16 & 0 & 689 & 2295 & 1526 & 95.38 \\
Ren's[39] & 9373 & 614 & 13 & 0 & 599 & 2455 & 1439 & 89.94 \\
Zhang's[42] & 9416 & 572 & 12 & 0 & 565 & 2022 & 1257 & 78.56 \\
Kanso's[44] & 9431 & 557 & 12 & 0 & 656 & 2391 & 1364 & 85.25 \\
Teh's[45] & 9969 & 31 & 0 & 0 & 575 & 2390 & 1379 & 86.81 \\
Wang's[46] & 9359 & 626 & 15 & 0 & 655 & 2046 & 1367 & 85.44 \\
This paper & 9495 & 505 & 0 & 0 & 685 & 1972 & 1253 & 78.31 \\
\hline
\end{tabular}

\section{Conclusion}

In this paper, we propose a circular shift based chaotic hash algorithm with variable parameters. We exploit piecewise linear chaotic map and one-way coupled map lattice to produce initial values and variable parameters. Circular shifts are introduced to improve the randomness of hash values. We evaluate the proposed hash algorithm in terms of distribution of the hash value, sensitivity of the hash value to slight modifications of the original message and secret keys, confusion and diffusion properties, robustness against birthday and meet-in-the-middle attacks, collision tests, analysis of speed, randomness tests, flexibility, computational complexity, and the results demonstrate that the proposed algorithm has strong security strength. Compared with the existing chaotic hash algorithms, our algorithm shows moderate statistical performance and better speed, randomness tests, and flexibility.

\section{Acknowledgements}


This work was supported by the National Natural Science Foundation of China [grant numbers 61402380, 61528206, 61672119]; the Natural Science Foundation of CQ CSTC [grant number cstc2015jcyjA40044]; the Fundamental Research Funds for the Central Universities [grant number XDJK2015B030]; U.S. National Science Foundation [grant numbers CNS-1253506 (CAREER), CNS-1618300]; and the Opening Project of State Key Laboratory for Novel Software Technology [grant number KFKT2016B13].

\section{References}

[1] Li Y, Xiao D, Deng S. Hash function construction based on the chaotic look-up table with changeable parameter. Int J Mod Phys B 2011;25:3835-3851.

[2] Tsudik G. Message authentication with one-way hash functions. ACM SIGCOMM Comput Commun Rev 1992;22:29-38.

[3] Rompel J. One-way functions are necessary and sufficient for secure signatures.

Proceedings of the 22th annual ACM symposium on Theory of computing; 1990: 387-394.

[4] Wang X, Feng D, Lai X, Yu H. Collisions for hash functions MD4, MD5, HAVAL-128 and RIPEMD. Cryptology ePrint Archive, Report 2004/199.

[5] Wang, X., Yin, Y.L., Yu, H.: Finding collisions in the full SHA-1. In: Advances in Cryptology-CRYPTO 2005, Lecture Notes in Computer Science, 2005;3621:17-36.

[6] Liang J, Lai X. Improved collision attack on hash function MD5. J Comput Sci Technol 2007;22(1):79-87. 
[7] Sasaki Y, Naito Y, Kunihiro N, Ohta K. Improved collision attacks on MD4 and MD5. IEICE T Fund Electr 2007;90-A(1):36-47.

[8] Mendel F, Nad T, Schlaffer M. Improving local collisions: New attacks on reduced SHA-256. In: Advances in Cryptology-EUROCRYPT 2013, Lecture Notes in Computer Science, 2013; 7881: 262-278.

[9] Stevens M. New collision attacks on SHA-1 based on optimal joint local-collision analysis. In: Advances in Cryptology-EUROCRYPT 2013, Lecture Notes in Computer Science, 2013;7881: 245-261.

[10]Wong KW. A combined chaotic cryptographic and hashing scheme. Phy Let A 2003;307:292-298.

[11] Amin M, Faragallah OS, El-Latif AAA. Chaos based hash function (CBHF) for cryptographic applications. Chaos Solitons Fractals 2009;42(2):767-772.

[12] Li Y, Xiao D, Deng S. Secure hash function based on chaotic tent map with changeable parameter. High Technol Lett 2012;18(1):7-12.

[13] Liu J, Wang X, Yang K, Zhao C. A fast new cryptographic hash function based on integer tent mapping system. J Comput 2012;7(7):1671-1680.

[14] Wang Y, Du M, Yang D, Yang H. One-way hash function construction based on iterating a chaotic map. In: Proceedings-CIS Workshops 2007, 2007 International Conference on Computational Intelligence and Security Workshops, 2007:791-794.

[15] Maqableh M, Samsudin AB, Alia MA. New hash function based on chaos theory (CHA-1). Int J Comput Sci Netw Secur 2008;8(2):20-26. 
[16] Jiteurtragool N, Ketthong P, Wannaboon C, San-Um W. A topologically simple keyed hash function based on circular chaotic sinusoidal map network. In: International Conference on Advanced Communication Technology, 2013:1089-1094. [17] Zhang Q, Zhang H, Li Z. One-way hash function construction based on conservative chaotic systems. In: 5th International Conference on Information Assurance and Security, 2009; 2: 402-405.

[18]Akhavan A, Samsudin A, Akhshani A. Hash function based on piecewise nonlinear chaotic map. Chaos Solitons Fractals 2009;42:1046-1053.

[19]Li Y, Xiao D, Deng S, Han Q, Zhou G. Parallel hash function construction based on chaotic maps with changeable parameters. Neural Comput Appl 2011;20(8): $1305-1312$.

[20]Xiao D, Liao X, Deng S. Parallel keyed hash function construction based on chaotic maps. Phys Lett A 2008;372:4682-4688.

[21]Xiao D, Liao X, Wang Y. Improving the security of a parallel keyed hash function based on chaotic maps. Phys Lett A 2009;373:4346-4353.

[22]Kanso A, Ghebleh M. A fast and efficient chaos-based keyed hash function. Commun Nonlinear Sci Numer Simul 2013;18:109-123.

[23]Nouri M, Khezeli A, Ramezani A, Ebrahimi A. A dynamic chaotic hash function based upon circle chord methods. In: 2012 6th International Symposium on Telecommunications, 2012: 1044-1049.

[24]Akhavan A, Samsudin A, Akshani A. A novel parallel hash function based on 3D chaotic map. EURASIP J Adv Signal Process 2013;(1):1-12. 
[25]Deng S, Li Y, Xiao D. Analysis and improvement of a chaos-based hash function construction. Commun Nonlinear Sci Numer Simul 2010;15(5):1338-1347.

[26]Alvarez G, Montoya F, Romera M, Pastor G. Cryptanalysis of dynamic look-up table based chaotic cryptosystems. Phys Lett A 2004;326(3):211-218.

[27] Arumugam G, Praba VL, Radhakrishnan S. Study of chaos functions for their suitability in generating message authentication codes. Appl Soft Comput 2007;7(3): 1064-1071.

[28] Li C, Wang S. New one-time signature scheme based on improved chaos hash function. Comput Eng Appl 2007;43(35):133-136.

[29] Guo W, Wang X, He D, Cao Y. Cryptanalysis on a parallel keyed hash function based on chaotic maps. Phys Lett A 2009;373(36):3201-3206.

[30] Xiao D, Peng W, Liao X, Xiang T. Collision analysis of one kind of chaos-based hash function. Phys Lett A 2010;374(10):1228-1231.

[31] Wang S, Shan P. Security analysis of a one-way hash function based on spatiotemporal chaos. Chin Phys B 2011;20(9):090504-090507.

[32] Wang S, Li D, Zhou H. Collision analysis of a chaos-based hash function with both modification detection and localization capability. Commun Nonlinear Sci Numer Simul 2012;17(2):780-784.

[33] Bakhtiari S, Safavi-Naini R, Pieprzyk J. Keyed hash functions. In: Proceedings of the Cryptography: Policy and Algorithms. Lecture Notes in Computer Science, 1996;1029:201-214. 
[34]WangY, Liao X, Xiao D, Wong K. One-way hash function construction based on 2D coupled map lattices. Inf Sci 2008;178(5):1391-1406.

[35]Shannon CE. Communication theory of secrecy systems. Bell Syst Tech J 1949;28(4):656-715.

[36]Rivest R. The MD5 message-digest algorithm. IETF Network Working Group, RFC 1321;1992.

[36]Deng S, Li Y, Xiao D. Analysis and improvement of a chaos-based Hash function construction. Commun Nonlinear Sci Numer Simul 2010;15:1338-1347.

[37]Li Y, Deng S, Xiao D. A novel Hash algorithm construction based on chaotic neural network. Neural Comput Appl 2011;20:133-141.

[38]Li Y, Xiao D, Deng S. Keyed hash function based on a dynamic lookup table of functions. Inf Sci 2012;214:56-75.

[39]Ren H, Wang Y, Xie Q, Yang H. A novel method for one-way hash function construction based on spatiotemporal chaos. Chaos Solitons Fractals 2009;42(4): 2014-2022.

[40] Xiao D, Liao X, Wang Y. Parallel keyed hash function construction based on chaotic neural network. Neurocomputing 2009;72:2288-2296.

[41] Xiao D, Shih FY, Liao XF. A chaos-based hash function with both modification detection and localization capabilities. Commun Nonlinear Sci Numer Simul 2010;15:2254-2261.

[42] Zhang J, Wang X, Zhang W. Chaotic keyed hash function based on feedforward-feedback nonlinear digital filter. Phy Let A 2007;362:439-448. 
[43] Zhang H, Wang X, Li Z, Liu D. One way hash function construction based on spatiotemporal chaos, Acta Physica Sinica 2005;54(9):4006-4011.

[44]Kanso A, Ghebleh M. A structure-based chaotic hashing scheme. Nonlinear Dyn 2015;81(1):27-40.

[45] Teh JS, Samsudin A, Akhavan A. Parallel chaotic hash function based on the shuffle-exchange network. Nonlinear Dyn 2015;81(3):1067-1079.

[46] Wang Y, Wong KW, Xiao D. Parallel hash function construction based on coupled map lattices. Commun Nonlinear Sci Number Simulat 2011;16:2810-2821.

[47]Luo Y-L, Du M-H. One-way hash function construction based on the spatiotemporal chaotic system. Chin Phys B 2012;21(6):060503.

[48]Chenaghlu MA, Jamali S, Khasmakhi NN. A novel keyed parallel hashing scheme based on a new chaotic system. Chaos Solitons Fractals 2016;87:216-225.

[49]Li Y, Ge G, Xia D. Chaotic hash function based on the dynamics S-Box with variable parameters. Nonlinear Dyn 2016;84(4):2387-2402.

[50]Li D, Zhang XP, Hu YT, Yang, YY. Adaptive synchronization between two fractional-order chaotic systems with uncertain parameters. J Southwest Univ (Natural Science Edition) 2016;37(11):1-8.

[51] NIST. Secure Hash Standard, http://csrc.nist.gov/CryptoToolkit/tkhash.html/; 2001 [accessed 15.01.22].

[52] Yi X. Hash function based on chaotic tent maps. IEEE Trans Circuits Syst II, Exp Briefs 2005;52:354-357. 
[53]Guo XF, Zhang JS. Keyed one-way hash function construction based on the chaotic dynamic S-Box. Acta Physica Sinica 2006;55:4442-4449.

[54]Rukhin A, Soto J, Nechvatal J, Smid M, Barker E, et al. A Statistical Test Suite for the Validation of Random Number Generators and Pseudo Random Number Generators for Cryptographic Applications (Version STS-2.1, NIST Special Publication $800-22$ rev1a) http://csrc.nist.gov/publications/nistpubs/800-22-rev1a/SP800-22rev1a.pdf/; 2010 [accessed 15.07.19].

[55]Marsaglia G. The Marsaglia random number CDROM including the DIEHARD battery of tests of randomness, http://stat.fsu.edu/pub/diehard/; 1996 [accessed 15.03.07].

[56]Walker J. ENT - A pseudorandom number sequence test program, http://www.fourmilab.ch/random/; 1993 [accessed 15.05.22].

[57] L'Ecuyer P, Simard R. TestU01: A C Library for Empirical Testing of Random Number Generators. ACM Trans Math Software 2007;33(4):Article 22,1-40.

[58] Akhshani A, Akhavan A, Mobaraki A, Lim S-C, Hassan Z. Pesudo ranom number generator based on quantum chaotic map. Commun Nonlinear Sci Numer Simulat 2014;19(1):101-111.

[59] Johnson S, Kotz NL. Discrete distributions. Boston: Houghton Mifflin Company; 1969. 